\title{
Sense and sensitisation: in vitro testing for hepatotoxic drug hypersensitivity
}

Over 30 years ago, small lymphocytes were observed to transform into larger, more granular cells and undergo proliferation in the presence of antigen in vitro. ${ }^{1}$ This rapidly formed the basis of a laboratory test to detect sensitised lymphocytes. ${ }^{2}$ In this system, mononuclear cells are separated from a patient's peripheral blood and cultured in medium in the presence of the suspected allergen. Monocytes are the major antigen presenting cells, although antigen presentation may also be performed, albeit to a lesser extent, by B cells. A positive result is most easily demonstrated by lymphocyte proliferation (usually measured by the uptake of tritiated thymidine) rather than by morphological transformation. The ratio of counts per minute in the presence of antigen compared with a control culture in its absence is expressed as the stimulation index. If this value is greater than a cut-off level determined in a set of healthy controls, then the test is considered positive, and evidence of the presence of sensitised lymphocytes. These cells are predominantly long-lived memory $\mathrm{T}$ cells ${ }^{3}$ identified by the surface expression of the CD45RO isoform and certain activation markers and adhesion molecules such as CD11a/CD18 (LFA-1), which accounts for their rapid reactivation on re-exposure to antigen.

The diagnosis of drug related liver damage is essentially one of exclusion, supported by clinical features such as the known patterns of drug reactions, the temporal association between ingestion of the drug and the time to liver damage, and by the response to drug withdrawal. In practice, the diagnosis is relatively clear although where a patient is taking several drugs or where the clinical picture is atypical, diagnosis of a drug reaction may be problematic. Hence, a simple confirmatory laboratory test would be of considerable value not only in making a positive diagnosis of hepatotoxic drug hypersensitivity but would also avoid potentially lethal inadvertent re-challenge with the drug, and unnecessary investigations. Unfortunately, the lymphocyte proliferation test-although apparently straightforward - has been shown to be unreliable under these circumstances, with positive results demonstrated in as few as $30 \%$ of cases. ${ }^{4}$ Indeed, on consideration of current theories of the mechanisms of drug induced immune mediated liver damage, it is remarkable that such a highly simplified in vitro test should ever be positive.

Very few drugs are directly hepatotoxic and most require metabolism to induce liver damage. Metabolites of those that initiate a specific immune response are capable of covalent binding to cellular or plasma constituents ${ }^{6}$ and subsequently undergo intracellular processing and surface presentation. For instance, antibodies against erythromycin are expressed on hepatocyte surface membranes. ${ }^{7}$ Not only may this result in drug epitopes being recognised, but alteration of the carrier molecule may lead to T cell receptor binding to self determinants and the generation of an autoimmune process. ${ }^{8}$ In the liver, reactive electrophilic and free radical metabolites, created by hepatic detoxifica- tion pathways, are capable of such binding to or alteration of intracellular protein, hence the liver specificity of many drug induced immune reactions. Indeed, in halothane induced hepatotoxicity, antibodies can be demonstrated to trifluoroacylated peptides that are produced following P450 oxidation and binding of the intermediate metabolite. ${ }^{9}$ Moreover, where the drug product binds to the oxidising enzyme itself, autoantibodies to the cytochrome P450 may be detected-as seen with the LKM2 antibodies (reacting with cytochrome P450 2C9) associated with tienilic acid hepatitis. ${ }^{9}$ In vitro evidence also supports such a premise. ${ }^{10}$ Tsutsui and colleagues managed to isolate CD4+ $\mathrm{T}$ cell clones that proliferated on exposure to the sensitising drug only in the presence of a partially purified liver protein preparation. ${ }^{11}$ In addition, the sensitivity of the lymphocyte stimulation test can be augmented by using serum from healthy volunteers that have ingested a test dose of the drug instead of using a crude preparation of the molecule itself. ${ }^{12}$ Although it is tempting to speculate that this is due to the hepatic production of reactive metabolites, it could equally be accounted for by binding to plasma proteins. The relatively small number of patients that demonstrate drug induced hypersensitivity compared with those exposed to the agent could be explained in this model by genetic variation in detoxification pathways. It may also be that a degree of direct toxic hepatocellular damage may be required to expose drug epitopes that are located intracellularly, and the antigen may be presented by class II HLA on Kupffer cells that have ingested hepatocyte fragments.

The standard lymphocyte proliferation test is not liver specific. However, production of reactive metabolites may be possible in vitro by lymphocytes ${ }^{12}$ which have been shown to express some of the relevant enzymes required. Immune responses are fundamentally dependent on the efficacy of antigen presentation, and the type of reaction engendered may be altered by molecular cross talk between the antigen presenting cell and the T cell. It is also surprising, therefore, that blood monocytes are capable of substituting for this activity in the liver. This is indeed the major factor limiting reproducibility in this test, which has been shown to depend critically on the number of lymphocytes and antigen presenting cells present in each well and the concentration and solubilisation of the drug. ${ }^{12}$ That the in vitro test does produce positive results without the requirement of liver specific factors might suggest that hepatic biotransformation of drugs is not as important as previously thought, and that the liver is targeted due to its ability to concentrate substances as a result of its anatomical position and "first pass" effect, and the expression of "homing" adhesion molecules on memory $\mathrm{T}$ cells limits the reaction to the organ of its induction. ${ }^{13}$

Notwithstanding the difficulties outlined above, Maria and Victorino in this issue (see page 534) describe their considerable experience of the lymphocyte proliferation 
test in 95 patients with clinically suspected hepatotoxic drug hypersensitivity seen over the past 12 years. With two relatively minor modifications to the test- the use of "ex vivo" antigens, and the addition of indomethacin to the culture medium - a sensitivity of as high as $88 \%$ was achieved. Activated monocytes in culture are capable of producing prostaglandins that have immunomodulatory properties, which may be abrogated by the use of a cyclooxygenase inhibitor. ${ }^{14}$ The resulting increase in lymphocyte proliferation seems to more than double the sensitivity of the test, and its mechanism is confirmed by the lack of response in cases where non-steroidal agents were also the suspected drugs. In addition, the authors have presented some data to suggest that the production of prostaglandin by a patient's cells in vitro was associated with an earlier remission and more benign clinical course in vivo. They hypothesise that this is due to a skewing of the cytokine profile from a Th1 to a Th2 pattern by inhibition of interferon- $\gamma$ secretion and a resultant decrease in Th1 supported cell mediated cytotoxicity. Prostaglandin E also seems to increase expression of interleukin- 5 and promote IgE isotype switching ${ }^{15}$; however, no increase in eosinophilia could be shown in these patients. This finding also raises the future possibility of immunotherapeutic intervention in severe hepatotoxicity with delayed response to drug withdrawal.

Of the patients in the study, $70 \%$ who had positive proliferation test results did not show any of the typical features of hypersensitivity such as a rash, fever or eosinophilia. An alternative explanation for the high frequency of positive stimulation responses in vitro in this study may be that the sensitised lymphocytes detected may be produced as a secondary effect of direct hepatotoxicity and may not necessarily indicate the presence of clinically significant immune mediated damage. Unlike the skin patch test, the lymphocyte proliferation test is flawed by not having an effector arm, and ideally in vitro testing of liver cell cytotoxicity would be required to confirm the immune mechanism of injury. Indeed, it is possible to demonstrate the presence of sensitised lymphocytes in patients who are clinically non-responders to the allergen by adding cytokines such as interferon- $\gamma$ and interleukin-1a to the lymphocyte culture along with prostaglandin inhibition. ${ }^{16}$ Interestingly, the four highest lymphocyte stimulation indexes in the current study were in patients with prolonged cholestasis due to pyritinol. In the presence of cholestasis, macrophage secretion and circulating levels of some cytokines are increased, in particular tumour necrosis factor $\alpha(\mathrm{TNF} \alpha) \cdot{ }^{17} \mathrm{By}$ stimulating antigen presenting cells, increasing secretion of other cytokines such as interferon- $\gamma$ and inducing expression of adhesion and activation markers, the presence of raised levels of $\mathrm{TNF} \alpha$ in vivo could potentially augment a borderline positive result of dubious significance, although no such effect has been demonstrated by addition of this cytokine to the culture medium in vitro. ${ }^{16}$ The authors of the current study did, however, control for confounding factors such as unrelated liver disease and found no positive stimulation responses to a range of drugs being taken by these patients (the baseline proliferation of lymphocytes under these conditions is not recorded). In addition, stimulation responses were not seen to other drugs being taken by patients with a positive proliferation test to one agent. However, temporarily high lymphocyte proliferation indexes have been reported by other investigators to one drug in the presence of a cholestatic hypersensitivity reaction to another, ${ }^{19}$ and suggests that under certain circumstances the test may detect sensitised lymphocytes that are not responsible for the induction of an immune mediated response. Finally, as indicated earlier, the diagnosis of drug related liver damage is one of exclusion.

Although simple in design, methodological difficulties may confound the application of the lymphocyte proliferation test in routine practice. Despite undoubted potential benefit, widespread clinical uptake of this test awaits confirmation of its specificity and reproducibility in the hands of less experienced investigators.

JEREMY WOODWARD

JAMES NEUBERGER

The Liver Unit,

Queen Elizabeth Hospital,

Birmingham B15 2TH, UK

Correspondence to: Dr J Neuberger.

1 Mills JA. The immunologic significance of antigen induced lymphocyte transformation in vitro. F Immunol 1966; 97: 239-47.

2 Caron GA, Sarkany I. Lymphoblast transformation in sulfonamide sensitivity. Br 7 Dermatol 1965; 77: 556-9.

3 Brattig NW, Diao G-J, Berg PA. The specificity of the lymphocyte transformation test in a patient with hypersensitivity reactions to Pyrazolone compounds. A 10-week follow-up study before and after re-challenge. Eur 7 Clin Pharmacol 1988; 35: 39-45.

4 Berg PA, Becker EW. The lymphocyte transformation test-a debated method for the evaluation of drug allergic hepatic injury. $f$ Hepatol 1995; 22: $115-8$

5 Warrington RJ, Tse KS. Lymphocyte transformation studies in drug hypersensitivity. Can Med Assoc F 1979; 120: 1089-94.

6 Parker CW. Hapten immunology and allergic reactions in humans. Arthritis Rheum 1981; 24: 1024-36.

7 Gomez-Lechon MJ, Carrasquer J, Berenguer J, Castell JV. Evidence of antibodies to erythromycin in serum of a patient following an episode of acute drug-induced hepatitis. Clin Exp Allergy 1996; 26: 590-6.

8 Pessayre D. Role of reactive metabolites in drug-induced hepatitis. 9 Hepatol 1995; 23 (suppl 1): 16-24.

9 Deleve LD, Kaplowitz N. Mechanisms of drug-induced liver disease. Gastroenterol Clin North Am 1995; 24: 787-810.

10 Hertl $M$, Jugert F, Merk HF. CD8+ dermal $T$ cells from a sulphamethoxazole-induced bullous exanthem proliferate in response to drug-modified liver microsomes. Br f Dermatol 1995; 132: 215-20.

11 Tsutsui H, Terano Y, Sakagami C, Hasegawa I, Mizoguchi Y, Morisawa S. Drug-specific $\mathrm{T}$ cells derived from patients with drug-induced allergic hepatitis. F Immunol 1992; 149: 706-16.

12 Maria VAJ, Pinto L, Victorino RMM. Lymphocyte reactivity to ex-vivo drug antigens in drug-induced hepatitis. $\mathcal{F}$ Hepatol 1994; 21: 151-8.

13 Willson RA. The liver. Its role in drug biotransformation and as a target of immunologic injury. Immunol Allergy Clin North Am 1991; 11: 555-73.

14 Victorino RMM, Maria VAJ, Pinto LA. Evidence for prostaglandinproducing suppressor cells in drug-induced liver injury and implications in
the diagnosis of drug sensitisation. Clin Exp Immunol 1992; 87: 132-7.

15 Phipps RP, Stein SH, Roper RL. A new view of prostaglandin E regulation of the immune response. Immunol Today 1991; 12: 349-51.

16 De Gruijl TD, Moore JJ, De Vries E, Von Blomberg-Van Der Flier BME, Fonk JCM, Scheper RJ. Augmentation of antigen-specific lymphoproliferative responses in vitro by biological response modifiers. Clin Exp Immunol 1994; 96: 535-40.

17 Puntis MC, Jiang WG. Plasma cytokine levels and monocyte activation in patients with obstructive jaundice. $\mathcal{F}$ Gastroenterol Hepatol 1996; 11: 7-13.

18 Scott-Conner CE, Grogan JB. The pathophysiology of biliary obstruction and its effect on phagocytic and immune function. I Surg Res 1994; 57: 316-36.

19 Hofer T, Becker EW, Weigand K, Berg PA. Demonstration of sensitised lymphocytes to trimethoprim/sulfamethoxazole and ofloxacin in a patient with cholestatic hepatitis. F Hepatol 1992; 15: 262-3. 